\title{
The cost-greenhouse gas emission nexus for water distribution systems including the consideration of energy generating infrastructure: an integrated conceptual optimization framework and review of literature
}

\author{
Christopher S Stokes*, Angus R Simpson and Holger R Maier
}

\begin{abstract}
The increased release of greenhouse-gas (GHG) emissions associated with human activities causing climate change is one of the most significant problems faced by human-kind. Water distribution systems (WDS), whilst providing an essential service to society, are responsible for the generation of significant amounts of GHGs. In response, the minimization of GHG emissions associated with WDSs has become a research focus. In this paper, a critical review of previous research is provided, summarizing research progress and highlighting research needs in this emergent and important area. This is done within the context of the water distribution system cost-emissions nexus (WCEN) conceptual framework, which is a novel conceptual framework that considers the interaction between all components required to accurately evaluate the costs and greenhouse gas (GHG) emissions associated with water distribution systems (WDSs) in an integrated fashion. Key findings from this review indicate that future research should (1) include the use of time-dependent emissions factors (EFs), which would allow the scheduling of pumps at times of lower emissions intense energy to be considered; (2) include the modeling of seasonally variable water demands; (3) include greater consideration of the hydraulic simulation process, such as using seasonal extended period simulations; (4) include greater consideration of the management of pumping operations at the design stage, instead of solely focusing on changes in infrastructure design to reduce costs and GHG emissions; (5) include consideration of the effects that external policies, such as carbon taxes and present value discounting, have on the trade-offs between costs and GHG emissions.
\end{abstract}

Keywords: Water distribution system; Greenhouse gas emissions; Multi-objective optimization; Sustainability; Hydraulic simulation; Water-energy nexus

\section{Introduction}

The increased release of greenhouse-gas (GHG) emissions associated with human activities causing climate change is one of the most significant problems faced by human-kind (Stokes et al. 2012). Greenhouse gas (GHG) releases through human-related activities have been identified as a major cause of human-induced climate change. The importance of mitigating climate change by reducing GHG emissions has been widely recognized by

\footnotetext{
* Correspondence: christopher.stokes@adelaide.edu.au

School of Civil, Environmental and Mining Engineering, University of
} Adelaide, Adelaide 5005, Australia the scientific, commercial and political sectors. Water distribution systems (WDSs) provide an essential service to modern cities. However, they also contribute significantly to the release of GHG emissions through activities related to their construction and operation, especially when pumping operational energy is sourced from fossil fuel electricity generation sources. WDSs are also complex systems, with many different design and operational options being available to a decision maker. Thus, it is often impractical or even impossible for a decision maker to evaluate and consider the combination of all available options. As such, optimizing the design and operation of 
WDSs via the use of optimization algorithms has become a popular way of considering these many available options. In order to evaluate the performance of the many potential design and operation combinations during evolutionary algorithm based optimization processes, WDSs must be modeled (i.e. simulated). However, the modeling of WDSs can be computationally expensive. As such, simplifications are often made during the modeling process in order to reduce both the problem complexity and the computational time required to evaluate each solution in the optimization process (in the case where evolutionary algorithms or similar metaheuristics are used). This can include simplifications to the decision variables, such as limiting the types and number of options considered; simplifications to input data, such as replacing actually time-dependent input information with steady-state or approximate values; and simplifications to the simulation process, such as hydraulically simulating a limited number of water demand scenarios compared to what will be encountered during real-life operations (Stokes et al. 2012). The optimization of costs associated with water distribution systems has been covered extensively in the past three decades (Wu et al. 2010a). As such, simplifications made to the modeling of WDSs have been well established. Consideration of optimizing WDSs for the minimization of GHG emissions has only occurred more recently. Commonly, GHG emissions (both capital emissions and operational emissions from fossil fueled electricity sources) have been optimized along with costs by using multi-objective (MO) optimization algorithms. As such, modeling simplifications applied when evaluating costs are also applied when evaluating GHG emissions. These simplifications have the potential to affect the possible solutions and their corresponding evaluations. In addition, the primary focus of optimization has been on the selection of WDS infrastructure design options (e.g. pipe sizes and pump types). Only limited consideration has been given to the impact of pump operational management, interactions between water supply infrastructure and energy generating infrastructure and how policy drivers may affect the optimal trade-offs between cost and GHG emissions. Therefore, there remains a need to review the current literature considering the optimization of WDSs for the minimization of GHG emissions in order to establish what modeling simplifications have been made and to identify gaps in current modeling and evaluation processes. In order to achieve this, a conceptual framework is required to identify and show the nexus of modeling elements that can impact on the optimization of costs and GHG emissions associated with WDSs. Additionally, this conceptual framework should include consideration of energy generating infrastructure that affects pumping operational GHG emissions, as well as policy drivers that can impact the trade-offs between costs and
GHG emissions associated with WDSs. Such a framework was first presented by Stokes et al. (2012). As such, the objectives of this paper are as follows:

1. To develop a conceptual framework, based on the framework presented by Stokes et al. (2012), that identifies and shows the interactions between the various modeling elements that have an impact on WDS cost and GHG emissions optimization, including those from energy generating infrastructure, in an integrated fashion.

2. To review existing literature considering the minimization of GHG emissions associated with WDSs in the context of the proposed conceptual framework in order to identify the research gaps with respect to the simplification of the modeling processes and future research required to address these gaps.

The water distribution system cost-emissions nexus (WCEN) conceptual framework (Objective 1), is presented in Water distribution cost-emissions nexus (WCEN) conceptual framework. The evaluation of existing literature in the context of the WCEN conceptual framework (Objective 2) is presented in Review of methods used for GHG emissions reduction associated with water distribution systems, leading to the identification of current research gaps and future research directions required to progress this field of research (Summary and conclusions and Recommendations for future research).

\section{Water distribution cost-emissions nexus (WCEN) conceptual framework}

The WCEN conceptual framework (Figures 1 and 2) is based on the similarly named framework presented by Stokes et al. (2012). While not an analytical tool itself, the WCEN conceptual framework represents the nexus of elements required to accurately model and evaluate costs and GHG emissions when optimizing the design and operation of a WDS. The conceptual framework is separated into four distinct components (Figure 1). These include an infrastructure component (WDS and electricity generation infrastructure), options component (design and operations of the WDS), analysis component (simulation and evaluation), and government policy subcomponent, each of which consists of a number of related elements. The components are linked to one another to represent the flow of information through the system. A list of components and sub-components of the conceptual framework is given in Table 1. In addition, the framework also consists of a simulation dynamics component (Figure 2), as the most appropriate simulation duration and number of simulations performed can have a significant impact on accuracy and 


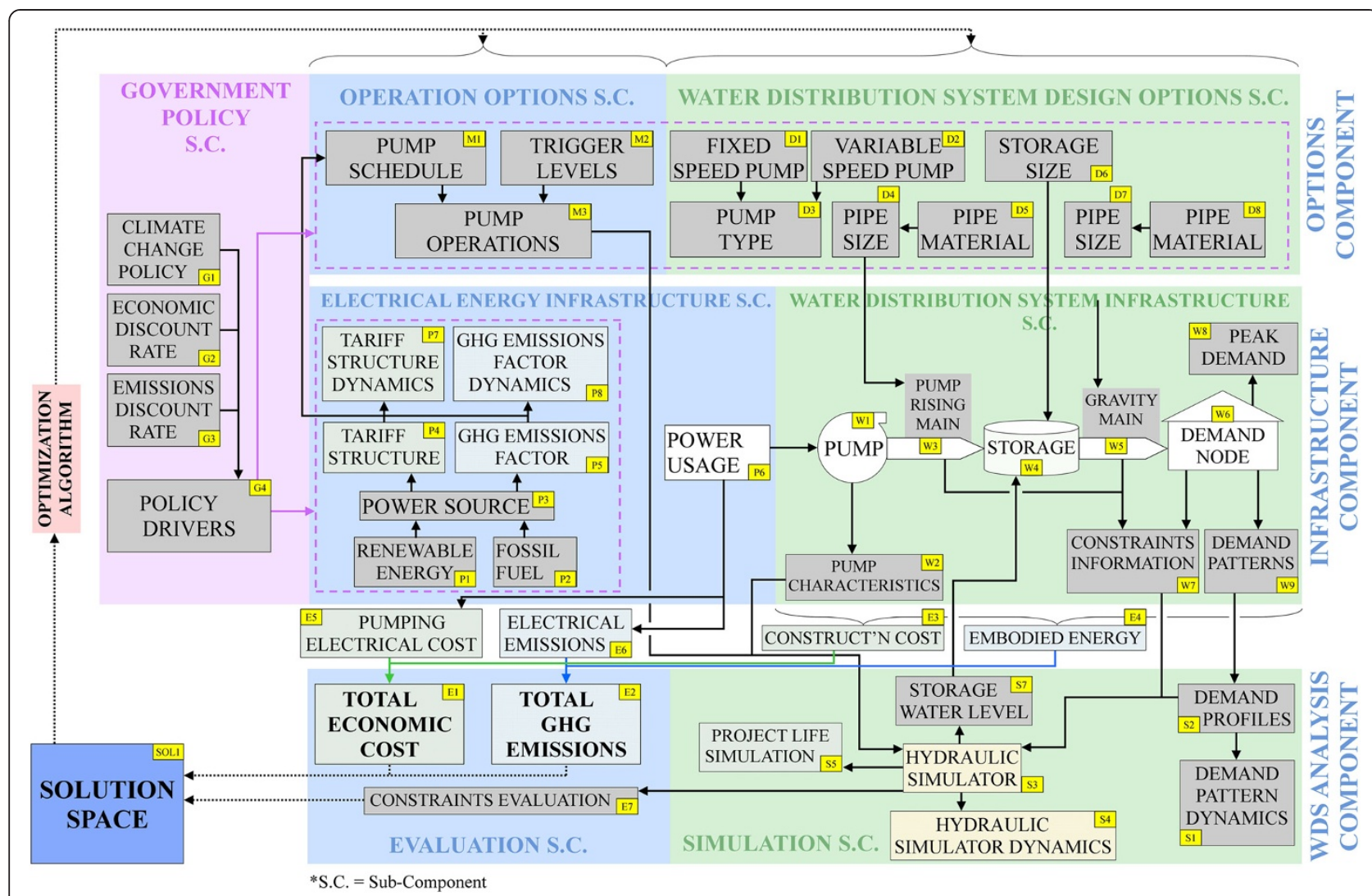

Figure 1 The water distribution system cost-emissions nexus framework (modified from Stokes et al. (2012)).

computational efficiency, and are likely to be different for the evaluation of costs and GHG emissions. The various sub-components of the WCEN conceptual framework are described in detail in the subsequent sections.

\section{Infrastructure component}

In order to obtain accurate estimations of the costs and GHG emissions when optimizing the design and operation of a WDS, it is important to consider the real-world infrastructure that is being modelled. The infrastructure component within the WCEN conceptual framework represents this real-world infrastructure. Two critical infrastructure types are important to consider. These include the WDS being modeled, as represented by the WDS infrastructure sub-component, and the sources of generation of electricity being used by pumps during the operation of WDSs, as represented by the electrical energy generating infrastructure subcomponent. While simplifications to both systems are required, each system's critical aspects, as related to the conceptual framework's purpose, should be retained.

Modeling of the WDS infrastructure is used to represent the physical WDS elements that allow water to be supplied from sources to consumers. An accurate representation of the critical elements of the actual WDS is required if costs and GHG emissions are to be estimated accurately. These elements are represented within the WDS infrastructure sub-component, and include the pumps that supply water to the system [W1 - See Figure 1]; the pump rising mains [W3] that connect the pumps to the distribution pipe network; the water storage systems [W4], which can include either reservoirs or tanks; the gravity mains that distribute water from water storages to the demand nodes [W5]; and the demand nodes, which represent the consumer demands placed on the WDS [W6]. Water demand patterns [W9] of the WDS being modeled are used within the hydraulic simulation process to consider the realworld water demands. A water demand profile [S2] can represent multiple water demand patterns for different demand node requirements (e.g. residential, commercial and industrial). A combination of multiple water demand patterns can also be used to represent different water demand scenarios, such as different seasons in a year. While peak and average water demand flows are commonly used when simulating a WDS, it can be important to consider a range of operational conditions in order to obtain the most accurate estimate of operational costs and GHG emissions. Additionally, it can also be important to consider exceptional water demand circumstances, such 


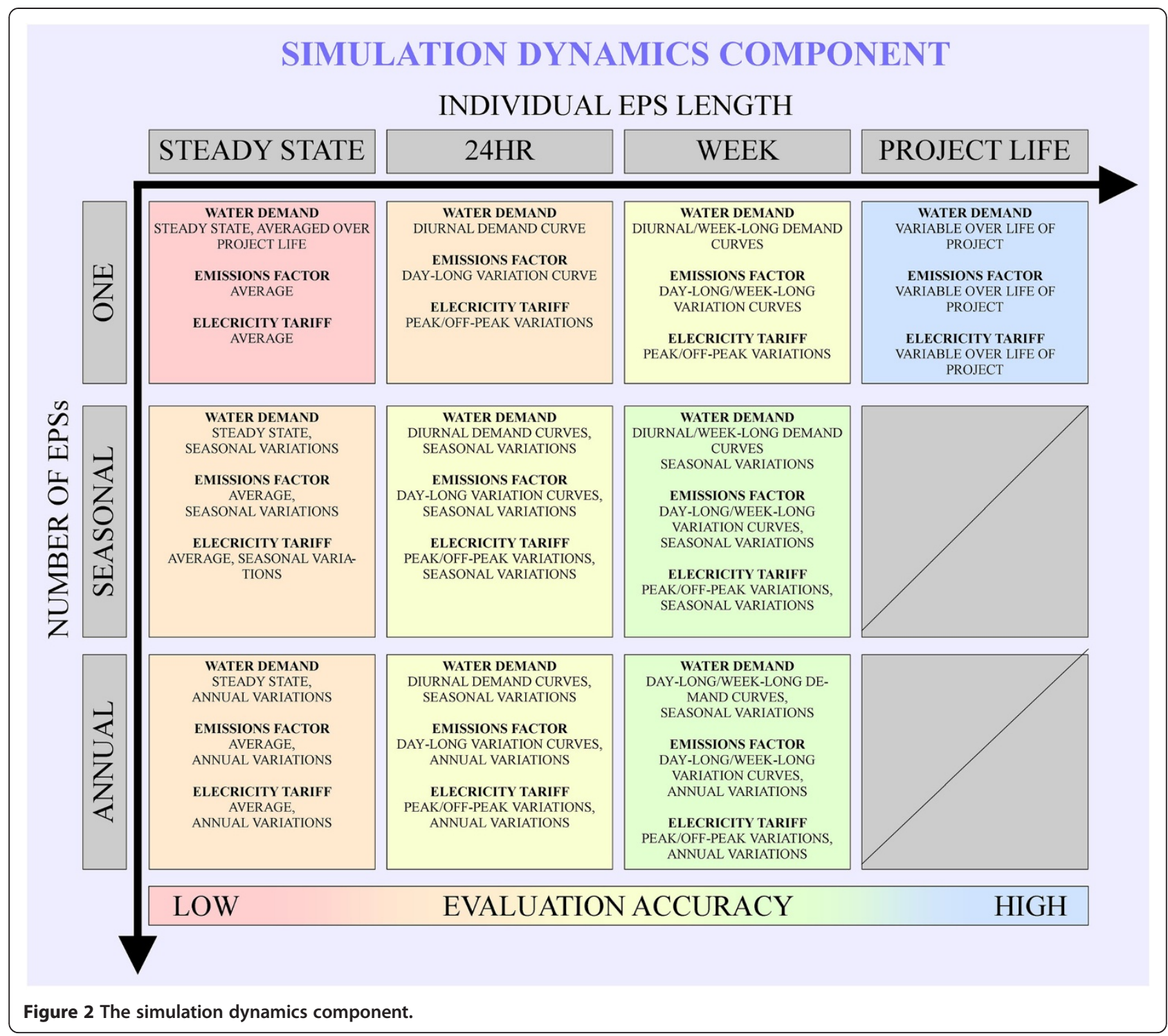

Table 1 Components and sub-components, water distribution system cost-emissions nexus framework

\begin{tabular}{cc}
\hline Component & Sub-component (SC) \\
\hline Options component & Operation options SC \\
& WDS Design options SC \\
Government policy SC
\end{tabular}

Infrastructure component Electrical energy infrastructure SC

WDS infrastructure SC

Government policy SC*

WDS analysis component

Simulation SC

Evaluation SC

*While the Government Policy sub-component is associated with both the Options and Infrastructure components, it is discussed separately from these components in the text. as fire loadings and pipe breakages. As water demands drive the system hydraulics, an accurate representation of both water demands and the physical infrastructure can help to obtain an accurate estimation of the pumping operational energy required to meet the demands. Additionally, accurate representation of the physical infrastructure is important if design related costs and GHG emissions are to be accurately estimated. Other aspects of a WDS, such as infrastructure maintenance and replacement, miscellaneous running costs (e.g. electricity for lighting at pump stations) and labor costs are not usually able to be included as part of the hydraulic simulation and are therefore not represented by the WDS infrastructure sub-component of the conceptual framework presented in this paper.

The electrical energy infrastructure sub-component represents the elements of electricity generation and supply 
infrastructure that are required to accurately evaluate pumping operational costs and GHG emissions associated with a WDS. The cost of electricity for pumping is commonly calculated by using an electricity tariff, which is represented by a tariff structure [P4]. The tariff structure dynamics [P7] represent the different possible tariff structures, such as flat rate or peak/off-peak rates. In order to accurately estimate the cost of pumping operational energy consumption, it is important to consider the variability in electricity tariffs during each day and/or week, as well as possible seasonal and annual variability. Pumping operational GHG emissions can be calculated by considering the generation rate and emissions factors of individual generators feeding into the grid. As such, both renewable [P1] and fossil fuel (non-renewable) [P2] generation types are represented in the conceptual framework. In order to accurately estimate the overall emissions factor [P5] of the electricity supplied to the WDS, the amalgamation of all individual generators supplying into the grid, represented as the electrical source [P3], should be considered. The use of emissions factors is represented by the emissions factors dynamics [P8]. Emissions factors can range from the use of a single, average value, to the use of multiple emissions factors used to represent the change in emission intensities over the period of a day, between each month/season in a year, or between each year over the operational life-span of the WDS. As a WDS is just one of many users consuming electricity from a grid, careful consideration should be given to how emissions factors associated with the consumption of electricity by the WDS [P5] are calculated (e.g. whether emissions factors values consider all generated electricity, or only the generation of electricity used by the WDS). While the consideration of how emissions factor values are calculated is beyond the scope of this paper, the application of the emissions factor values must also be carefully considered. The way in which emissions factors are used can affect the evaluation of emissions.

\section{Options components}

In order to find solutions of minimized costs and GHG emissions when optimizing the design and operation of a WDS, it is important to consider the options available to decision makers. These options are represented within the options component by two sub-components; the water distribution system design options (WDS design options) sub-component and the operation options sub-component.

The WDS design options sub-component is used to represent the options related to the design of the hydraulic infrastructure. Design phase considerations commonly include the selection of sizes of pipes, storage tanks/reservoirs and pumps, and are generally assumed to be fixed after the construction (or redevelopment/rehabilitation) of the system. Chosen pump types [D3], both variable-speed pumps (VSPs) [D2] and fixed-speed pumps (FSPs) [D1], pipe sizes [D4, D7], material types [D5, D8] and water storage sizes [D6] can significantly affect design costs and GHG emissions associated with the products themselves and operational costs and GHG emissions, through their effect on system hydraulics. While design costs may be evaluated from pricing information gained from commercial sources, design GHG emissions must be calculated directly from the materials used. Embodied energy is commonly used to calculate these GHG emissions. A widely used definition of embodied energy has been given by Treloar (1994).

Options available for the operational management of WDSs are represented by the operation options subcomponent. Pumping operations can be explicit (using pump scheduling) and/or implicit (using storage trigger levels). Pump scheduling [M1] can be used to control the timed status and speed of pumps, while trigger levels [M2] can be used to control storage levels. Chosen control options are represented as pump operation information [M3]. This information can be used to represent operational scenarios via the use of hydraulic simulation [S3], allowing pumping operational energy consumption to be calculated. While average conditions can be used to estimate pumping operational energy consumption, more accurate estimations can be achieved by considering multiple operational scenarios.

\section{Water distribution system analysis components}

In order to obtain more accurate trade-offs between costs and GHG emissions when optimizing the design and operation of a WDS, it is important to consider both the simulation and evaluation options available. To do this, the water distribution system analysis component of the conceptual framework uses two sub-components; the simulation sub-component, which represents the operational simulation of the WDS, and the evaluation sub-component, which represents the evaluation of costs and GHG emissions associated with the WDS. Evaluation of costs and GHG emissions can be achieved both directly from the design options, represented by the options component, and indirectly through operational simulation, represented by the simulation sub-component. The evaluation of objective functions using infrastructure design and hydraulic simulation information has been used extensively within the field of WDS optimization.

Hydraulic simulation [S3] is used to evaluate both design constraint satisfaction and objective function performance of each developed solution. Project life simulation [S5] represents the simulation of the WDS over the life of the project, including consideration of both construction and operation phases. Project life simulation can incorporate both infrastructure design information (from the options component) and information gained 
from the hydraulic simulation. Information outputted from an extended period simulation (EPS) can include the storage levels [S7], pipe flows and node pressure information at each time-step, which can be used for constraint evaluation [E7], and pump electrical energy consumption [P6] used for operational evaluation purposes. Hydraulic simulation requires water demand profiles [S2], pump characteristics (pump and efficiency curves) [W2] and pump operation information [M3]. Constraint information [W7], such as water balance and node pressure requirements, is used for the evaluation of constraints. Demand profiles can be used to simulate water demand changes over different seasons and years, as represented by the demand pattern dynamics [S1], to better represent the true nature of water demands. The hydraulic simulator requires a representation of the physical system; this information is commonly a simplified model of the real-life WDS, as represented by the WDS infrastructure sub-component, and includes design options information, as represented by the WDS design options sub-component. The total hydraulic simulation length can be controlled by modifying the EPS length and the number of different EPSs used (e.g. used for changes of input data values, such as emissions factors and water demands, over different months/seasons or years), which are represented by the hydraulic simulator dynamics [S4].

Evaluation of each objective function, namely total life cycle economic cost [E1] and total life cycle GHG emissions [E2], is represented by the evaluation subcomponent. This sub-component is also used to represent constraint evaluation [E7], which is used to penalize designs that violate user-defined design constraints (such as node pressure and water balance violations). Design and operational information represented by both the water distribution system and electrical energy infrastructure sub-components is used to evaluate the fitness of each solution. Infrastructure construction costs [E3] and pumping electrical costs [E5] are used to evaluate total life cycle economic costs. GHG emissions from electrical energy consumption [E6] and from embodied energy (Treloar 1994) associated with infrastructure construction [E4] are used to evaluate total life cycle GHG emissions.

\section{Simulation dynamics components}

The simulation dynamics component (Figure 2) is used to represent the temporal dynamics of the hydraulic simulation. This includes representation of the number of EPSs (e.g. for different seasons) and the length of each EPS. The dynamics of the water demand model, the emissions factor model and the electricity tariff model are represented as variables used to adjust the level of accuracy achieved by the simulation process. The EPS dynamics are represented as a function of the other dynamic variables; the requirements for the number of EPSs and length of each EPS are dependent on how the water demands, emissions factors and tariffs are to be modeled. The EPS dynamics represent the transition of input data accuracy into hydraulic simulation and evaluation accuracy. In order to accurately estimate costs and GHG emissions, input data must be accurate, which in turn requires appropriate hydraulic simulations in order to account for this accuracy (e.g. using a 24 hour EPS to account for the use of diurnal water demands). In this way, each variable can be modeled to replicate the reallife operational environment as accurately as possible. However, this way of simulating the WDS requires a single EPS running over the length of the project life, which is computationally expensive and would usually be time prohibitive for use with optimization. This would also require future water demands, emissions factors and electricity tariffs to be known for the entire length of the project life, which would not be possible when modeling such complex systems.

In order to achieve accurate evaluation, particularly for electrical energy consumption, which is cumulative over the lifespan of the WDS as discussed earlier, while minimizing the time taken to perform the optimization, a compromise must be made. The most common way of increasing simulation accuracy whilst minimizing computational expense is to use a single EPS, where short term (daily) changes to the water demand and tariff are modeled. However, this does not consider longer term changes, such as seasonal and yearly variations. In order to accurately estimate operational costs and GHG emissions, it is important to consider both short and long term variations by considering different EPS lengths and numbers of EPSs used. While four different EPS lengths and three different numbers of EPSs are shown, other lengths and numbers of EPSs can also be used, depending on the requirements of the modeled demand, emissions factor and tariff data used.

\section{Government policy sub-components}

Policies and governance external to the control of a water utility can have a significant effect on both the design and operation of a WDS and the evaluation of its associated costs and GHG emissions. These policies are represented by the government policy sub-component. Three policy types are focused on, including climate change policy [G1], economic discount rate policy [G2] and emissions discount rate policy [G3]. These policies can significantly affect the operational costs and GHG emissions of a WDS when accumulated over longer time-periods. Therefore, it is important to consider the effects of policies over the entire life of a WDS, including both design and operational phases. This component has been included to highlight the importance of being 
able to consider the effects of policy on the optimal design and operation of a WDS.

\section{Review of methods used for GHG emissions reduction associated with water distribution systems}

In this section, papers that have focused on the minimization of GHG emissions associated with water distribution systems using formal optimization approaches are reviewed in the context of the WCEN conceptual framework introduced in the previous section, discussing the achievements that have been made within this field and the aspects that require further research. Additional papers that focus on the minimization of GHG emissions associated with WDSs from an analysis or simulation perspective are also included in the review. In total, thirty one journal papers, eighteen conference papers and one report have been included in the review (see Additional file 1: Table S1). While the WCEN conceptual framework focuses on the minimization of GHG emissions, papers considering energy reduction have also been included. It should be noted that while many papers that focus on the reduction of costs associated with WDSs exist, only those explicitly considering the reduction of either energy (within the context of reducing environmental impact) or GHG emissions are reviewed in this paper. The components of the WCEN conceptual framework considered in each paper are summarized in Additional file 1: Table S1 and discussed in detail in the subsequent sections.

\section{Consideration of options}

The papers that have considered aspects represented within the options component of the WCEN framework are presented in Additional file 1: Table S1. As can be seen, the most widely used options associated with the design of WDSs are pipe sizing [Additional file 1: Table S1, Column C7] (Pr3, Pr4, Pr5, Pr10, Pr11, Pr12, Pr15, Pr16, Pr23, Pr24, Pr25, Pr26, Pr27, Pr31, Pr32, Pr33, Pr34, Pr35, Pr36, Pr39, Pr40, Pr41, Pr42, Pr43, Pr44, Pr45, Pr46, Pr47, Pr48, Pr49-See Additional file 1: Table S1) and the selection of pipe material type [C6] (Pr3, Pr5, Pr10, Pr11, Pr12, Pr15, Pr16, Pr23, Pr24, Pr25, Pr26, Pr27, Pr31, Pr32, Pr33, Pr34, Pr35, Pr36, Pr39, Pr40, Pr41, Pr42, Pr43, Pr44, Pr45, Pr46, Pr47, Pr48, Pr49). Other options, such as storage tank size and location [C5] (Pr4, Pr5, Pr19, Pr23, Pr24, Pr26, Pr33, Pr40, Pr44) and pump type selection [C3] (Pr2, Pr10, Pr27, Pr33, Pr39, Pr40, Pr41, Pr42, Pr43, Pr44, Pr45, Pr46, Pr47, Pr48, Pr49) were also used. Operational management options (pump scheduling [C1] and trigger levels [C2]) were not used as frequently (Pr5, Pr9, Pr14, Pr31, Pr33, Pr34, Pr36, Pr46).

Trade-offs can occur between the design and operational phases which can be affected by the options chosen for each phase. For example, a major trade-off can occur between the minimization of pipe sizes to minimize capital costs/GHG emissions and the minimization of pump energy consumption to minimize operational costs/ GHG emissions. Similarly, trade-offs can occur between the objectives of minimizing costs and GHG emissions. For example, similar to electricity tariffs, the emissions intensity of electricity is time-dependent. Therefore changing the time-of-use of pumps can alter both the GHG emissions and costs associated with the electricity consumed, even if the amount of electricity consumed does not change. If the rise and fall of emissions factors and electricity tariffs do not coincide, trade-offs will be seen between operational costs and GHG emissions. While these examples are easy to grasp, other trade-offs may be more implicit, requiring more thorough analysis in order to understand their causes and effects.

\section{Pipe size selection}

As can be seen in Additional file 1: Table S1, pipe size selection [C7] is the most common option considered. Twenty eight of the reviewed papers considered the pipe sizes used in a WDS. Twenty five of these used the pipe size option as a decision variable for optimization, with twenty considering the reduction of GHG emissions. The majority of these showed a trade-off between construction and operational GHG emissions and while reduced pipe sizes also reduced GHG emissions associated with pipe construction, total GHG emissions (construction and operation) increased due to an increase in pumping energy required to overcome the higher friction losses of the smaller pipe sizes. However, some other interesting results were reported. Herstein et al. (2009a) (Pr25) showed that an increase in pipe size resulted in an increase in environmental impact (using the environmental index (EI) measurement). The use of larger pipes in this system allowed more water to be pumped to the storage tank instead of directly to the demand node. However, as the tank was located further away from the pump location, this resulted in greater energy losses, and thus an increase in energy usage, resulting in the reported increase in EI value. Results from $W u$ et al. (2010b) (Pr45) showed a trade-off between construction and operational GHG emissions that result in an optimal design that uses a relatively small pipe size (compared to the choices available). This is probably due to a low demand, with larger pipe sizes resulting in a relatively low pump energy usage reduction compared to the increase in construction emissions associated with the additional material required for larger pipes. Dandy et al. (2008) (Pr11) used multi-objective optimization to reduce the pipe costs and energy of a gravity fed system. As there was no operational energy expenditure, the lowest energy solution corresponded to the lowest pipe embodied energy solution. 
From the reviewed literature, it is clear that a trade-off exists between construction and operational GHG emissions due to the sizes of pipes used in WDSs. A general trend of reducing pipe sizes (lower construction GHG emissions) resulting in increased pump energy requirements (higher operational GHG emissions) has been noted. However, other factors such as system layout, system hydraulic capacity and consumer water demands directly affect the point at which an optimal trade-off is found. While the area of WDS GHG emissions optimization is relatively new, the majority of research focused on the option of pipe size selection, with results showing a clear benefit of considering GHG emissions when sizing pipes for both WDS design and upgrade scenarios.

\section{Pipe material selection}

Twenty eight of the reviewed papers considered the type of material used for the construction of pipes. The majority of these used the concept of embodied energy to evaluate the environmental impact of pipe material type selection [C6]. Ambrose et al. (2002) (Pr3) considered the specific values for pipe embodied energy for different material types. While embodied energy values vary between each material type, it was noted that the quoted embodied energy value for a specific material type is also dependent on the level of detail used during the calculation of the embodied energy. While many pipe material types are available, the option of material type was commonly limited to either ductile iron cement mortar lined (DICL), polyvinyl chloride (PVC) or polyethylene (PE) pipes (Pr10, Pr11, Pr23, Pr32, Pr36), though Du et al, (2013) (Pr12) also compared these along with concrete, reinforced concrete and cast iron pipe materials. However, many papers considered the selection of only one material type (Pr4, Pr5, Pr24, Pr25, Pr26, Pr27, Pr31, Pr32, Pr33, Pr39, Pr40, Pr41, Pr42, Pr43, Pr44, Pr45, Pr46, Pr47, Pr48, Pr49). Wu et al. (2008a) (Pr40) noted that while DICL has a relatively low embodied energy value when compared to that of PVC and PE based pipes, it also has a relatively high unit mass, which can also affect a pipe's associated GHG emissions and needs to be considered. Ambrose et al. (2002) (Pr3) showed that despite the apparent benefit of DICL, it contained an embodied energy up to five times that of PVC and PE based pipes when the unit mass and hydraulic performance of each pipe type was considered. Du et al. (2013) (Pr12) found that ductile iron had the greatest (worst) global warming potential (GWP, based on embodied energy analysis) for smaller pipe diameters, while PVC had the greatest GWP for larger pipe diameters due to the pipe wall thickness used for these larger diameters. Despite high production energy demands and carbon dioxide emissions, concrete pipes were found to have the lowest (best) GWP between pipe diameters of $102 \mathrm{~mm}$ and $1219 \mathrm{~mm}$. Case study results by MacLeod et al. (2010) (Pr32) and Roshani et al. (2011) (Pr36) both showed little difference in GHG emissions of optimal designs using PVC or DICL pipes, although the construction costs of PVC pipes were considerably lower than those of DICL pipes. Dandy et al. (2006) (Pr10) considered both PVC and DICL pipe materials for the energy reduction optimization of a WDS and found that the optimal design used only PVC pipes. While Roshani et al. (2011) (Pr36) and MacLeod et al. (2010) (Pr32) only evaluated GHG emissions associated with operations, Dandy et al. (2006) (Pr10) evaluated energy associated with both capital (construction) and operations.

While only six of the reviewed papers focusing on optimization considered multiple material type choices for the construction of pipes, different studies showed different pipe materials to be beneficial for the reduction of GHG emissions. The work by Ambrose et al. (2002) (Pr3) showed a large difference in the embodied energy of DICL and PVC material types. PVC pipes have been shown to have a lower embodied energy value per unit length of pipe, which would suggest that they also have a lower environmental impact with respectively lower GHG emissions over DICL pipes. This finding was also shown by Dandy et al. (2006) (Pr10). However, the literature also suggested that pipe material type has little effect on the hydraulics of a WDS, resulting in little difference in operational GHG emissions. This suggests that while the difference in hydraulic performance (i.e. frictional losses) between material types may only be small, the differences in embodied energy values of the pipes can have a substantial effect on the overall GHG emissions associated with a particular design.

\section{Pump type selection}

Of the reviewed papers, fourteen considered the option of pump type selection during optimization. Pump type selection [C3] has been used in conjunction with pipe size selection by $W u$ et al. (2010a; 2010b; 2008a; 2008b; 2009; 2010c; 2012a; 2008c; 2010d; 2012b; 2013) (Pr39, Pr40, Pr41, Pr42, Pr43, Pr44, Pr45, Pr46, Pr47, Pr48, Pr49), Kang and Lansey (2012) (Pr27) and Marchi et al. (2014) (Pr33), while using multi-objective optimization to find the optimal trade-off between construction and operational GHG emissions. Additionally, Richardson and Hodkiewicz (2011) (Pr35), while not considering pump type selection per se, considered the effect of pump overhaul scheduling, and hence the trade-offs between pump replacement capital and loss of efficiency due to wear, on the minimization of cost and GHG emissions. This study showed that similar trade-offs exist between costs and GHG emissions when considering pump overhaul scheduling as when considering other more often used options, such as selecting pipe sizes and pump types. Wu et al. (2010c) (Pr46) and 
used trigger levels to control the operation of a pump over a 48-hour EPS, while Marchi et al. (2014) (Pr33) considered the use of both pump scheduling and trigger levels to control pump operations. These studies highlighted the importance of considering both pump type selection and pump operational management together. The other studies stated above used steadystate analysis without the use of pump operational management, with the range of GHG emissions corresponding to optimal solutions being far smaller than those obtained while incorporating pump operational management. The ability to reduce GHG emissions by considering pump type selection and pump operational management options together has not been considered in depth in the reviewed literature. However, results showed that this consideration may lead to further reductions in GHG emissions, and it is therefore recommended that this be further explored.

\section{Pump operational management}

Eight of the reviewed papers considered the use of pump operational management. Of these, seven used storage trigger levels [C2] to implicitly control pumps, while four considered the use of pump scheduling [C1] to explicitly control the time of operation. Ertin et al. (2001) (Pr14) and Ramos et al. (2011) (Pr34) used both pump schedules and trigger levels, comparing the energy efficiency of each management type. Ertin et al. (2001) (Pr14) showed that a $12.5 \%$ energy saving can be made by using pump scheduling instead of storage tank trigger levels. Conversely, Ramos et al. (2011) (Pr34) reported that while no pump electrical energy savings were made by using pump scheduling instead of trigger levels, operational costs can be significantly reduced by pumping at off-peak electricity times and hence reducing the average unit cost of consumed electrical energy. Trigger level options were also used for the purpose of reducing GHG emissions (Pr31, Pr33, Pr34, Pr36, Pr46) and energy usage (Pr9, Pr14). While literature considering operational management options has suggested a benefit to the consideration of pump operational management for the reduction of GHG emissions, little work has been undertaken to consider the effects of time-dependent operational factors, such as time-dependent emissions factors, on the optimal operational management of WDSs. However, as considering the time-dependency of electricity tariffs has been shown to help select operational management choices that reduce operational costs (Pr34), by extension, consideration of the timedependency of emissions factors could help to reduce operational GHG emissions. As pumps use the majority of consumed energy during WDS operation, careful consideration of pump control represents a possibility for further
GHG emissions reduction and therefore warrants further research.

\section{Infrastructure considerations Water distribution system complexity}

WDSs have been represented within the literature in different forms, from simple single pipe systems to complex, real-world networks. As can be seen from Additional file 1: Table S1, of the reviewed literature using multiobjective (MO) optimization and the objective of GHG emissions reduction, eleven examples used complex WDSs [C16], while fourteen of the reviewed papers used only simplified WDSs [C15] for case-studies. Simplified networks have been used for proof of concept and assessment of the impact of policy factors, such as discount rates. More complex networks were used for both initial design and system upgrade scenarios. Case-studies by Abadia Sanchez et al. (2008) (Pr1), Cabrera et al. (2010) (Pr9) and Filion et al. (2004) (Pr17) used simplified representations of WDSs for the purpose of system energy analysis. Ertin et al. (2001) (Pr14), Filion (2007; 2008) (Pr15, Pr16) and MacLeod and Filion (2011) (Pr31) used simplistic systems in order to analyze the effects of specific factors, such as pump scheduling, population density and urban form, on the energy usage and/or GHG emissions associated with a WDS. Herstein et al. (2009a) (Pr25) used a one pump, one tank and one demand node WDS in order to test the concept of the environmental impact index; used to rank a WDS based on several sustainability criteria, including the release of GHG emissions. This was later applied to an MO optimization problem using the Anytown WDS (Pr26), as described by Walski et al. (1987). Biehl and Inman (2010) (Pr5), Boulos and Bros (2010) (Pr7), Ektesabi et al. (2009) (Pr13) and Young (2010) (Pr50) discussed possible energy reduction and GHG emissions abatement strategies, and the considerations that need to be made when applying them to real-world systems. Ghimire (2010) (Pr21) and Ghimire and Barkdoll (2008; 2009; 2010) (Pr18, Pr19, Pr20) simulated a number of WDSs ranging in size and complexity, analyzing the effects of various factors on energy usage, such as pump power, storage tank parameters and water demands. Wu et al. (2013) (Pr49) optimized a South Australian WDS, among others, for the minimization of costs and GHG emissions and the maximization of hydraulic reliability. MacLeod et al. (2010) (Pr32) and Roshani et al. (2011) (Pr36) optimized the Amherstview, Canada, WDS as an upgrade problem, looking at the effect of pipe selection on GHG emissions, while Dandy et al. (2006) (Pr10) optimized the Anabranch rural WDS in Australia as a design problem, looking at the effect of pipe selection on capital and operational energy usage, with comparison to an original design, which focused on the reduction of capital and operational costs. 
In summary, there has been limited consideration of complex WDSs. While they were used for the simulation and analysis of energy usage and the analysis of GHG emissions, only eleven of the reviewed papers used complex systems in case-studies for the optimization of GHG emissions (Pr4, Pr5, Pr12, Pr23, Pr24, Pr26, Pr27, Pr32, Pr33, Pr36, Pr49). While simple case-studies have shown the benefits of considering GHG emissions, only the use of more complex case study systems will be able to show the feasibility of considering GHG emissions associated with real-world WDSs outside of the research arena. Therefore, further research should be undertaken in order to understand the implications of considering GHG emissions on more complex systems.

\section{Water demands}

Daily water demand patterns [C17], also known as diurnal curves, were incorporated by nineteen of the reviewed papers into the simulation and optimization of energy usage and GHG emissions associated with WDSs (Pr2, Pr4, Pr5, Pr8, Pr9, Pr14, Pr19, Pr21, Pr22, Pr23, Pr24, Pr25, Pr31, Pr32, Pr33, Pr34, Pr36, Pr46, Pr49). Diurnal curves have become a popular way to increase the accuracy of modeling the time-dependency of water demands seen in the real world. This time dependency has become an important part of modeling the cost of operating WDSs, especially with the consideration of peak/off-peak electricity tariffs, where it is not only the total time of pump operation that is important, but also the time of use. Ertin et al. (2001) (Pr14) demonstrated a reduction in energy usage of $12.5 \%$ when considering pump time of use. This was done by careful consideration of storage tank levels, which required the use of diurnal curves to accurately simulate the change in tank levels over time. While the majority of literature considering GHG emissions opted for the use of steady-state water demands, Herstein et al. (2009a) (Pr24, Pr25), MacLeod and Filion (2011) (Pr31) and Wu et al. (2010c) (Pr46) included the use of diurnal curves while using extended period simulations (EPSs) when evaluating operational energy usage. While not commonly in use, demand variations [C18] over extended periods of time, such as monthly, seasonal and annual variations, have also been incorporated. Alandi et al. (2009b) (Pr2) used monthly demand variations in order to evaluate pump energy usage for each month in the year and Filion et al. (2004) (Pr17) used demands that were assumed to increase on a decade by decade basis. The demand variations were used to consider the difference in system requirements at each stage of pipe replacement during the life of the system. Wu et al. (2012b) (Pr47) incorporated seasonal demand variations as a way of assessing the benefits of using variable speed pumps. In this study, the benefit of being able to reduce the pump's speed was seen by a reduction in frictional energy loss, which in turn equated to a reduction in GHG emissions.

Nineteen of the reviewed papers used diurnal water demand patterns as a consideration of the time-dependency of consumer demands. This is important, as it allows the time-dependency of real-life water demands to be represented more accurately. The time-dependency of water demands over longer time periods is still rarely used, with only two optimization papers considering this (Pr17, Pr47). However, as shown by Filion et al. (2004) (Pr17) and $W u$ et al. (2012b) (Pr47), considering longer term water demand variations can affect the choice of optimal design options. Consideration of water demand variability is important for the accurate analysis of GHG emissions, as a WDS is a demand driven system and thus this consideration can directly affect the energy usage requirements of the system. Water demands may change over the operational life of a WDS (e.g. diurnal changes, seasonal changes and/or yearly changes) and these changes must be incorporated in order to more accurately reflect actual energy consumption. In order to achieve greater accuracy, future research will need to incorporate longer-term water demand changes along with the shorter-term changes that are presently used.

\section{Electricity tariffs}

Single, average tariff values [C12] have been predominantly considered when analyzing the operational costs associated with WDSs (Additional file 1: Table S1). Of the reviewed papers, only four considered peak/ off-peak time-dependent tariffs [C13] (Pr6, Pr8, Pr33, Pr34). Biehl and Inman (2010) (Pr6) discussed the ways in which electricity is charged to the consumer, and suggested ways in which both energy usage and its associated costs can be reduced. Both time dependent charges, including peak and off peak tariffs, which charge for the actual amount of electricity used with a rate based on the time of usage, and time-independent charges, including demand charges, which charge for the highest demand reached over either the billing period, or a prescribed period of time, were considered. While a demand charge can account for $10-20 \%$ of a water utility's electricity costs, it is suggested that the majority of these costs can be attributed to tariff charges (Pr6). Ramos et al. (2011) (Pr34) showed that optimizing pump operations while considering peak/off-peak electricity tariffs can result in cost reductions by pumping during off-peak times. One study also looked at the effect of longer term changes to electricity costs. Wu et al. (2012a) (Pr48) used a fixed rate tariff, adjusted annually to model the effect of electricity price increases caused by the possible effects of carbon taxes and carbon trading schemes imposed on the electricity generation industry. 
While literature using peak/off-peak electricity tariffs has shown that consideration of the time-dependency of electricity tariffs can be used to reduce operational costs (Pr34), there has been little research to assess the effects of time-dependent tariffs on the trade-offs between costs and GHG emissions. Although tariffs are only used to calculate costs associated with electricity usage, the trade-offs that often occur between costs and GHG emissions mean that the accurate analysis of operational costs is an important part of analyzing this trade-off. As with GHG emissions, operational costs are accumulated over the life of a WDS and as such, both the short-term and long-term time-dependencies of electricity tariffs must be considered if these costs are to be assessed accurately.

\section{Greenhouse gas emissions factors}

As can be seen from Additional file 1: Table S1, all of the reviewed papers which used emissions factors used single, average GHG emissions factors [C10] instead of considering short-term (e.g. diurnal) emissions factor variations. The only consideration of time-dependent emissions factors [C11] in the reviewed literature was by Roshani et al. (2011) (Pr36) and Wu et al (2012a; 2013) (Pr48, Pr49). In these studies, emissions factors were assumed to reduce annually, due to an increase in the proportion of renewable energy sources for electricity generation. However, short-term (e.g. daily) variations of emissions factors were not considered. Within the literature, there has been little discussion of the short-term variability of emissions factors, which considers the varying contribution of different generation types for different demand loads during the day. However, similar to electricity tariffs, emissions factors can vary over shorter (e.g. daily) time periods. Similar to the effect of electricity tariffs on costs (Pr34), these changes to emissions factors have the potential to affect the optimal operation of pumps when considering the minimization of GHG emissions. GHG emissions are accumulated over the lifetime of a WDS's operation. As such, the time of use of electricity generated from fossil fuel sources has the potential to considerably alter the GHG emissions associated with the operation of a WDS. For WDS optimization, there lies a potential to find reduced GHG emissions operational strategies by considering the impact of timedependent GHG emissions factors. However, this has not been studied thus far.

\section{Sources of electrical energy generation}

While the analysis of pump energy usage was widely considered, only seven of the papers reviewed considered the source of electricity [C9] consumed by pumping activities (Pr25, Pr26, Pr30, Pr34, Pr36, Pr38, Pr48). These papers commonly accounted for the types of electricity generation by considering their associated emissions factors. This consideration allows the emissions factor for a specific electricity generation region to be evaluated, allowing for increased accuracy when evaluating GHG emissions. Stokes and Horvath (2005) (Pr38) used life cycle analysis (LCA) to evaluate the energy use and GHG emissions for two case-study WDSs in California. GHG emissions were evaluated for multiple activities throughout the life of the WDSs; including through the use of electricity for pumping, which was calculated considering the mix of electricity generation types for the state of California. Lundie et al. (2006) (Pr30) also used LCA to evaluate the environmental impacts of Sydney Water's activities, including the WDS used to supply the city. In this study, both conventional and alternative power sources, including the combustion of biosolid remains from wastewater treatment, were considered. Ramos et al. (2011) (Pr34) compared operational management optimization while considering different power sources, including from the electricity grid, a water turbine used to recover energy normally lost through a pressure reducing device and a wind turbine used to provide renewable energy generation. The results of this study concluded that using renewable energy (in the form of a wind turbine) can significantly reduce GHG emissions, as significantly less electricity is sourced from the electricity grid. Herstein et al. (2009a; Lundie et al. 2004) (Pr25, Pr26) included the consideration of electricity generation sources during the optimization of case-study WDSs, in which system cost and environmental impact were evaluated. The environmental impact objective used considers several factors, including air pollution and non-renewable resource depletion, associated with the use of electricity. The consideration of electricity generation source was used in the evaluation of these factors, where the type of generation impacts the amount of pollution and resource depletion.

Pump energy usage [C14] is often calculated as part of the analysis of a WDS. While the energy usage of a pump is generally considered, the consideration of where this energy has come from is often overlooked. This is important if the GHG emissions associated with the usage of electricity are to be calculated more accurately. However, only seven of the reviewed papers considered different sources of electricity generation (Pr25, Pr26, Pr30, Pr34, Pr36, Pr38, Pr48). While consideration was given to the location of electricity generation sources (generally on a regional basis), little research has been conducted into the influence of time on these sources. A WDS can operate over many decades, with GHG emissions associated with its operation being accumulated over this period. Because of this, accurate calculation of these GHG emissions will require consideration of the source of electricity generation in terms of both location 
and time. In order to accurately estimate GHG emissions, greater consideration needs to be given to the sources of electricity generation in order to increase the accuracy of GHG emissions analysis.

\section{Water distribution system analysis considerations Extended period simulations}

Of the reviewed papers that used hydraulic simulation, thirty six used either single steady state or extended period simulation [C19], of no more than 96 hours in length, to evaluate energy use over the projected lifespan of the WDS. The majority of these have not considered variable lengths of EPS and the effect this can have on the accuracy of evaluation. However, two papers have discussed EPS length [C21]. Cabrera et al. (2010) (Pr10) used two EPS lengths during a WDS energy audit; one day and one year, with energy inputs and outputs being evaluated using both simulation lengths. The proportion of total input/output energy associated with each source/consumer was compared over the different EPS periods. Hernandez et al. (2010) (Pr22) also used various EPS lengths while conducting a WDS energy audit. In this case, short-term and long-term EPSs of one day and one month, respectively, were used.

The use of multiple hydraulic simulations [C22] can also help to improve the accuracy of evaluation. For example, the simulation of different demand patterns over multiple seasons within a year can be used to reflect the changing demands that occur in the real world; however, this requires a separate EPS for each demand pattern, which will increase the computational time required to run an optimization algorithm. Most of the reviewed literature has used a single hydraulic simulation in order to evaluate pump energy requirements. Exceptions to this include Alandi et al. (2009b) (Pr2), who simulated multiple demand scenarios for each month in the year; and $W u$ et al. (2012b) (Pr47), who simulated the use of both FSPs and VSPs over four demand scenarios to represent seasonal variation, using the demand variations to show the energy saving benefits of using VSPs over FSPs. Filion et al. (2004) (Pr18) also used multiple simulations for the purpose of analyzing multiple demand scenarios. Increases in demand were used at each system upgrade juncture, which require possible pipe size changes in order to fulfil hydraulic demands for the next maintenance period.

Few papers have considered the length and number of EPSs used to analyze the operation of a WDS. However, these constitute important considerations. As discussed in Simulation dynamics components, the use of water demand, electricity tariff and GHG emissions factor data that consider time-dependent variations will require simulations that encompass these time variations. Without considering these, the increased accuracy of the input data will not be translated into more accurate analysis. As such, research must consider the length and number of EPSs used, with consideration given to the requirements of the input data used.

\section{Government policy considerations Economic discounting}

As can be seen from Additional file 1: Table S1, twelve papers considered the effects of economic discounting [C28], using discount rates ranging from $1.4 \%$ to $10 \%$. Comparisons were also made between the results found by using different discount rates (Pr31, Pr32, Pr36, Pr39, Pr40, Pr41, Pr42, Pr43, Pr44, Pr45). Results commonly showed higher annual operating cost designs resulting from the use of higher discount rates. This result is expected, as a higher discount rate will place less value on future (operating) costs compared to present (construction) costs, resulting in a bias towards lower construction cost designs. This results in designs that require the use of more electrical energy for pumping requirements. The use of higher discount rates translates to greater pump energy requirements, with an associated increase in GHG emissions. The largest proportion of GHG emissions commonly results from electricity usage during operations. Reducing total GHG emissions can often be achieved by reducing operational GHG emissions, which has been seen with the use of lower discount rates. In practice, higher discount rates are applied to economic cost analyzes for water distribution systems ( $\mathrm{Pr} 43)$, however, the results shown within the reviewed literature would suggest that a lower discount rate should be applied to economic costs if importance is also to be placed on reducing GHG emissions.

Eleven of the sixteen papers which used optimization to reduce GHG emissions also considered the use of economic discount rates, which represents the majority of papers. Present value analysis (PVA), used to evaluate the present worth of future activities, is critical to the analysis of trade-offs between construction and operational costs, as the discount rate used can have a dramatic effect on the weighting given to operation. As such, sensitivity analyzes of economic discount rates will still be necessary in order to analyze these trade-offs in a robust fashion.

\section{Greenhouse gas emissions discounting}

While not as commonly considered as economic discounting, PVA was also applied directly to the evaluation of GHG emissions [C29] in nine of the reviewed papers. A discount rate of zero is often used for GHG emissions impact evaluation (Pr44), placing an equal weighting on present GHG emissions and those produced in the future. Use of positive discount rates was also suggested (reducing the value of future emissions), 
which reflects the belief that future technology will be able to better abate the impact of higher GHG emission concentrations in the atmosphere (Pr44). Of the reviewed papers that considered GHG emission discounting, the majority used a rate of zero. Wu et al. (2008c) (Pr41) used two discounting scenarios; economic costs and GHG emissions costs (using a carbon tax) were discounted at the same rate for the first scenario, while GHG emissions costs were given a zero discount rate in the second scenario. The results of this study show that the second scenario leads to results where a higher proportion of total costs are due to GHG emissions. Another study by $W u$ et al. (2010a) (Pr44) used the same scenarios as described above, while GHG emissions were discounted directly, however, a direct comparison between the two scenarios was not presented.

As with economic discount rates, the direct application of discount rates to GHG emissions is an important aspect of the analysis process. Trade-offs exist between construction and operational GHG emissions and also between costs and GHG emissions. As such, careful consideration needs to be given to the discount rates applied to GHG emissions. However, as discussed above, few studies have taken the effects of GHG emissions discounting into account. As with economic PVA, there remains a need to consider the effects of GHG emissions PVA with the use of sensitivity analyzes and the consideration of the effects different discount rates have on the trade-offs between the construction and operation phases, and the objectives of cost and GHG emission reduction.

\section{Carbon costing}

Carbon tax and carbon trading policies [C27] have been analyzed in six of the reviewed papers. This was done by applying a monetary cost to each unit of GHG emissions produced, including that from construction, calculated from embodied energy, and operation, calculated from electricity usage. Roshani et al. (2011) (Pr36) used three carbon tax scenarios as proposed by the Canadian National Round Table on the Environment and the Economy (2009), comparing optimization results for each. However, this study found little evidence that the use of a carbon tax will result in GHG emissions benefits, concluding that for the system upgrade problem considered, there was already adequate hydraulic capacity, suggesting that upgrading the system would do little to reduce pump energy requirements. MacLeod and Filion (2011) (Pr31) used the same carbon tax scenarios as Roshani et al. (2011) (Pr36), applied to a water transfer main design scenario. Results of this study showed that a larger pipe diameter was chosen for the two higher taxed scenarios when the lowest discount rate was used, resulting in fewer GHG emissions being produced during operation over the lifetime of the project. Wu et al. (2008c) (Pr41) applied five different carbon taxes to a WDS optimization problem. As with MacLeod and Filion (2011) (Pr31), a higher carbon tax showed some propensity to result in the selection of larger pipe diameters, thus reducing pump energy requirements. Wu et al. (2012a) (Pr48) used an increase in electricity costs to simulate the effect of a carbon trading scheme, with electricity tariffs increasing annually by a set percentage. Results from this study suggest that no significant GHG emissions reductions would be seen by considering higher electricity costs, as the use of higher electricity tariffs increased the operational cost of each design solution, however, it did not affect the order of the solutions.

The results from the above studies suggest that the use of carbon taxes and carbon trading schemes may help to reduce GHG emissions, however, there are other factors that need to be considered, which may also play a significant role in the choice of optimal solutions. These include the use of different discount rates, the emissions factors applied to the use of electricity and the impact of changing pipe sizes on a system's hydraulic capacity. While these studies have helped to recognise the benefits of carbon taxes and carbon trading schemes, more research is needed to understand what level of carbon tax and carbon costing is required to see optimal benefits in relation to the reduction of GHG emissions, and whether this can be applied to all cases or is casestudy specific.

\section{Summary and conclusions}

The rising level of GHG emissions within the atmosphere of the Earth is a common problem faced by human-kind, with no easy solutions yet to be discovered. As such, it is the responsibility of each sector of industry to help reduce their contribution of GHG emissions released into the atmosphere. Water utilities are no exception. Research into the GHG emissions associated with WDSs is a new, yet important field. There remain many aspects of GHG emissions reduction that are yet to be properly researched. The importance of the field, coupled with the responsibility of water utilities to reduce their carbon footprint, means that these areas should become a priority for future research efforts.

Water distribution systems (WDSs), whilst providing an essential service to modern cities, contribute significantly to the release of GHG emissions. Optimization has been used as a way to more efficiently design and operate WDSs by reducing both costs and GHG emissions. This paper has presented the WCEN conceptual framework (Water distribution cost-emissions nexus (WCEN) conceptual framework), a conceptual tool used to analyze the components which affect the costs and GHG emissions associated with WDSs, and has reviewed current literature which considers the use of formal 
optimization methods for the reduction of GHG emissions (and energy usage, which is linked directly to GHG emissions in most cases) associated with WDSs (Review of methods used for GHG emissions reduction associated with water distribution systems). The review of the selected papers has outlined gaps in the current literature, which are summarized in Recommendations for future research.

While not an analytical tool itself, the WCEN conceptual framework provides a representation of all the components required to accurately evaluate the GHG emissions and costs associated with WDSs. This includes the integration of electricity generation infrastructure, used to more accurately represent the factors affecting GHG emissions associated with electricity usage; the introduction of more accurate, time-dependent input data, including water demands, electricity tariffs and GHG emissions factors; the ability to modify the hydraulic simulation process to fit the requirements made by the use of more accurate input data; the analysis of outside policies such as present value discounting policy and carbon trading policy; and the integration of these aspects into one complete framework.

\section{Recommendations for future research}

The literature reviewed in this paper has shown the benefits of reducing climate change effects that have come with the explicit consideration of GHG emissions in the optimization of WDSs. While trade-offs often exist between costs and emissions, it has been shown that the consideration of GHG emissions does not need to be at the detriment to cost savings. While the reviewed literature has introduced the concept of evaluating the GHG emissions associated with a WDS, there is scope for improvements to be made in the field of WDS simulation and optimization. Improvements need to be made so that GHG emissions are evaluated with the same degree of accuracy as costs. Greater accuracy will be found by both improving the input data used and careful consideration of the modeling process. An increase in accuracy will not only allow solutions to be viewed with greater confidence, but will also allow better solutions to be found.

Based on the review of the fifty papers on the reduction of energy usage and GHG emissions associated with the construction and operation of water distribution systems considered in this paper, the following recommendations for future research are made.

1) Costs, associated with both the design and operation of WDSs, have been well considered within the literature. Similarly, GHG emissions associated with the design of WDSs have been well considered, both in terms of factors affecting design GHG emissions (e.g. embodied energy analysis) and the choices available to control design GHG emissions (e.g. choosing pipe diameters). However, GHG emissions associated with the operation of WDSs have been given little consideration beyond simplistic evaluation. While considerations of material types and their respective production methods have been made in order to accurately evaluate design GHG emission, similar accuracy has not been afforded to operational GHG emissions. Considering the sources of electricity used for pumping purposes is critical, as they can have a significant impact on the emissions intensity of electricity being consumed. Future research should focus on the consideration of the sources of electricity, so that operational GHG emissions can be evaluated as accurately as costs and design GHG emissions.

2) Consideration should be given to the timedependency of GHG emissions factors used for the evaluation of operational GHG emissions resulting from the operation of pumps. As discussed in Greenhouse gas emissions factors, current research predominantly treats emissions factors used to calculate GHG emissions as a single, average value. The sources of electricity (see recommendation 1) need to be considered if the time-dependency of emissions factors is also to be considered. However, as discussed in Sources of electrical energy generation, there is a lack of consideration of the source (s) of electricity used for pumping. Both of these gaps mean that the GHG emissions associated with electricity usage are not being accurately evaluated, with little consideration being given to both the time and place of electricity usage. In reality, emissions factors fluctuate with time and location according to the contribution of different generation types supplying to the electricity grid. As discussed previously, the time-variability of electricity tariffs has been successfully used to reduce the cost of WDS operations. Similar to this, the modeling of time-variability of emissions factors could not only increase the accuracy of operational GHG emissions evaluation, but could allow pump operational strategies to be explored, using potential times of low emissions energy as a way to reduce GHG emissions without the necessity of reduced energy consumption. While emissions factors may be difficult to accurately model due to the complex nature of the electricity generation industry, they may be modeled using similar ideas to those employed for water demands. These could include diurnal curves for hourly fluctuations through the day; multipliers used to adjust the peaks for different times of the year; and predictions for future 
increases/decreases over the coming years and decades.

3) If the time-dependency of emissions factors is to be considered, then it is also necessary to consider the time-dependency of water demands. As water demands affect the timing and magnitude of water requirements placed on the WDS, they can directly affect the energy requirements of pumps and as such, affect the optimal use of pumps. Additionally, as the driver for the entire system, the accuracy of modeling a WDS is dependent on the modeling accuracy of water demands. As discussed in Water demands, while diurnal curves are now widely used to model the variation in water demands over the length of a day, other demand variations have not been widely considered within the reviewed literature. As GHG emissions are accumulated over the life of a WDS, longer term variations, such as seasonal and annual variations, should be modeled in order to accurately simulate the effect that changing water demands have on the amount of GHG emissions produced over the operational lifetime of a system.

4) In order to benefit from the additional accuracy afforded by considering time-dependent emissions factors and water demands, the time-of-use of pumps also needs to be considered. Pumps can be controlled to both reduce energy usage through unnecessary friction losses due to high pipe velocities and to use electricity during low emissions factor times to reduce operational GHG emissions. However, pumps also need to be controlled so that the ever-changing water demands placed on the WDS are met, without storage tanks running empty or below a minimum acceptable level. As such, the complex task of operating pumps to minimize costs and GHG emissions is ideally suited to formal optimization techniques. However, as discussed in Pump operational management, little consideration has been given to pump operational management options for the reduction of GHG emissions associated with WDSs. As the majority of GHG emissions (in a pumped system) are commonly associated with the use of pumps, there exists an opportunity to further reduce GHG emissions by considering optimal operational management of pumps within WDSs.

5) As discussed in Extended period simulations, little consideration has been given to the hydraulic simulation processes used for the evaluation of GHG emissions. Few improvements in the simulation processes applied to WDSs (including simulation length and the number of simulations used) have been considered in the reviewed papers. If the use of more accurate information, such as time-dependent
GHG emissions factors and seasonal/annual water demand variations is to be considered, careful consideration of the simulation process is also required. The necessity to modify simulation practices when incorporating new input data has been highlighted in Figure 2, where the addition of input information complexity is matched against simulation requirements necessary to fully exploit the additional information. As such, if recommendations 1 to 4 are to be considered, it will also be necessary to further consider the requirements of the simulation processes used to evaluate operational costs and GHG emissions.

6) As discussed in Government policy considerations, government policies have been considered in the reviewed papers by including such factors as discount rates for both economic and GHG emissions discounting, and carbon pricing by considering carbon taxes and carbon trading schemes. While one or more of these factors have been included by thirteen of the sixteen papers that have used optimization to reduce GHG emissions, they have a significant effect on the evaluation of costs and GHG emissions. As such, it is important that policy factors are continually considered.

\section{Additional file}

Additional file 1: Table S1. Matrix representation of the reviewed literature (Basupi et al. 2013; Basupi et al. 2014; Bunn and Reynolds 2009; Herstein and Filion 2011; Kiselychnyk et al. 2009; Kumar and Karney 2007; Simpson 2008).

\section{Competing interests}

The authors declare that they have no competing interests.

\section{Authors' contributions}

CSS carried out development of the WCEN conceptual framework, review of literature and drafted the manuscript. ARS participated in the development of the WCEN conceptual framework and helped to draft the manuscript. HRM participated in the development of the WCEN conceptual framework and helped to draft the manuscript.

\section{Acknowledgements}

The authors would like to thank the anonymous reviewers for their valuable contributions to this paper. The authors would also like to thank the University of Adelaide and the Goyder Institute for Water Research for their financial support of this research.

Responsible editor: Michael Piasecki.

Received: 12 October 2013 Accepted: 25 March 2014

Published: 23 May 2014

\section{References}

Abadía Sánchez R, Rocamora Osorio C, Ruiz Canales A, Puerto Molina H, Melián Navarro A: Energy efficiency in irrigation: calculation approach in water distribution system. In Agricultural and Biosystems Engineering for a Sustainable World International Conference on Agricultural Engineering; 23-25 June. Hersonissos, Crete, Greece: European Society of Agricultural Engineers (AgEng); 2008:OP-1380. 
Alandi PP, Pérez PC, Álvarez JFO, Hidalgo MÁM, Martín-Benito JMT: Pumping selection and regulation for water-distribution networks. J Irrig Drain Eng 2005, 131:273-281

Ambrose MD, Salomonsson GD, Burn S: Piping systems embodied energy analysis. CSIRO Manufacturing and Infrastructure Technology: Highett, Australia; 2002

Basupi I, Kapelan Z, Butler D: Reducing life-cycle carbon footprints in the redesign of water distribution systems. J Water Climate Change 2013, 4:176-192.

Basupi I, Kapelan Z, Butler D: Reducing life-cycle carbon footprint in the (re) design of water distribution systems using water demand management interventions. Urban Water J 2014, 11:91-107.

Biehl WH, Inman JA: Energy optimization for water systems. J Am Water Works Assoc 2010, 102:50-55.

Boulos PF, Bros CM: Assessing the carbon footprint of water supply and distribution systems. J Am Water Works Assoc 2010, 102:47-54.

Bunn S, Reynolds $L$ : The energy-efficiency benefits of pump-scheduling optimization for potable water supplies. IBM J Res Dev 2009, 53:5.1-5.13.

Cabrera E, Pardo MA, Cobacho R, Cabrera E Jr: Energy audit of water networks. J Water Resour Plan Manag 2010, 136:669-677.

Dandy GC, Roberts A, Hewitson C, Chrystie P: Sustainability objectives for the optimization of water distribution networks. In Proceedings of 8th Annual Water Distribution Systems Analysis Symposium; August. Cincinnati, USA: American Society of Civil Engineers; 2006

Dandy GC, Bogdanowicz A, Craven J, Maywald A, Liu P: Optimizing the sustainability of water distribution systems. In 10th Annual Symposium on Water Distribution Systems Analysis; 17-20 August. Kruger National Park, South Africa: American Society of Civil Engineers; 2008

Du F, Woods GJ, Kang D, Lansey KE, Arnold RG: Life cycle analysis for water and wastewater pipe materials. J Environ Eng 2013, 139:703-711.

Ektesabi M, Moradi-Motlagh A, Abdekhodaee A: Energy and sustainability through integrated water network management. WIT Trans Ecol Environ 2009, 121:175-186.

Ertin E, Dean AN, Moore ML, Priddy KL: Dynamic optimization for optimal control of water distribution systems. In Applications and Science of Computational Intelligence IV; 2001:142-149.

Filion YR: Connections between population density, energy use, and GHG emissions in water networks. In CSCE 2007 Annual General Meeting and Conference; 6-9 June. Yellowknife: NWT; 2007:151-160.

Filion YR: Impact of urban form on energy use in water distribution systems. $J$ Infrastruct Syst 2008, 14:337-346.

Filion YR, MacLean HL, Am A, Karney BW, Asce M: Life-cycle energy analysis of a water distribution system. J Infrastruct Syst 2004, 10:120-130.

Ghimire SR: Relationship between pump horsepower and energy usage in municipal water networks. In World Environmental and Water Resources Congress. Providence, Rhode Island, USA: American Society of Civil Engineers; 2010:4180-4184.

Ghimire SR, Barkdoll BD: Effect of demand on energy use in municipal water distribution systems. In World Environmental and Water Resources Congress. Honolulu, Hawai'i: American Society of Civil Engineers; 2008.

Ghimire SR, Barkdoll BD: Impact of storage tanks on energy consumption in municipal water distribution systems. In World Environmental and Water Resources Congress. Kansas City, Missouri, USA: American Society of Civil Engineers; 2009:158-164.

Ghimire SR, Barkdoll BD: Sensitivity analysis of municipal drinking water distribution system energy use to system properties. Urban Water J 2010, 7:217-232.

Hernández E, Pardo M, Cabrera E, Cobacho R: Energy assessment of water networks, a case study. In Water Distribution System Analysis. Tuscon, AZ: American Society of Civil Engineers; 2010:1168-1179.

Herstein LM, Filion YR: Life-cycle assessment of common water main materials in water distribution networks. J Hydroinf 2011, 13:(3)346-357.

Herstein LM, Filion YR, Hall KR: Evaluating environmental impact in water distribution system design. J Infrastruct Syst 2009a, 15:241-250.

Herstein LM, Filion YR, Hall KR: EIO-LCA based multi-objective design of water distribution systems with NSGA-II. In Computing and Control in the Water Industry 2009 'Integrating Water Systems'; September 1-3. London, U.K: Taylor and Francis Group; 2009b:225-231.

Herstein LM, Filion YR, Hall KR: Evaluating the environmental impacts of water distribution systems by using ElO-LCA-based multiobjective optimization. J Water Resour Plan Manag 2011, 137:162-172.
Kang D, Lansey K: Dual water distribution network design under triple-bottom-line objectives. J Water Resour Plan Manag 2012, 138:162-175

Kiselychnyk O, Bodson M, Werner H: Overview of energy efficient control solutions for water supply systems. Kremenchuk, Ukraine: Transactions of Kremenchuk State Polytechnic University; 2009:40-46.

Kumar G, Karney B: Electricity usage in water distribution networks. In Electrical Power Conference. Montreal, Quebec, Canada: IEEE; 2007:97-102.

Lundie S, Peters GM, Beavis PC: Life cycle assessment for sustainable metropolitan water systems planning. Environ Sci Technol 2004, 38:3465-3473.

MacLeod SP, Filion YR: Issues and implications of carbon-abatement discounting and pricing for drinking water system design in Canada. Water Resour Manag 2011, 26:44-61.

MacLeod S, Roshani E, Filion YR: Impact of pipe material selection on greenhouse gas mitigation in water networks under uncertain discount rates and carbon prices. In Water Distribution System Analysis. Tuscon, AZ: American Society of Civil Engineers; 2010:1014-1027.

Marchi A, Salomons E, Ostfeld A, Kapelan Z, Simpson AR, Zecchin AC, Maier HR, Wu ZY, Elsayed SM, Song Y: The battle of the water networks II (BWN-II). J Water Resour Plan Manag 2014

National Round Table on the Environment and the Economy: Achieving 2050: A carbon pricing policy for Canada. Ottawa: Library and Archives Canada Cataloguing in Publication; 2009

Ramos HM, Kenov KN, Vieira F: Environmentally friendly hybrid solutions to improve the energy and hydraulic efficiency in water supply systems. Energy Sustain Dev 2011, 15:436-442.

Richardson SJ, Hodkiewicz MR: Modeling tool to support budgeting and planning decisions for pump overhauls. J Water Resour Plan Manag 2011, 137:327-334

Roshani E, MacLeod SP, Filion YR: Evaluating the impact of climate change mitigation strategies on the optimal design and expansion of the Amherstview, Ontario water network: a Canadian case study. J Water Resour Plan Manag 2011, 138:100-110.

Simpson AR: Selecting a discount rate for evaluating water distribution projects - The sustainability controversy. In Water Distribution System Analysis; August 17-20. Edited by Van Zyl K. American Society of Civil Engineers; 2008.

Stokes J, Horvath A: Life cycle energy assessment of alternative water supply systems. Int J Life Cycle Assess 2006, 11:335-343.

Stokes CS, Simpson AR, Maier HR: An improved framework for the modelling and optimisation of greenhouse gas emissions associated with water distribution systems. In 6th International Congress on Environmental Modelling and Software (iEMSs). Leipzig, Germany: International Environmental Modelling and Software Society; 2012.

Treloar GJ: Energy analysis of the construction of office buildings: Master of Architecture Thesis. Melbourne, Australia: Deakin University; 1994.

Walski TM, Brill JED, Gessler J, Goulter IC, Jeppson RM, Lansey K, Lee HL, Liebman JC, Mays L, Morgan DR, Ormsbee L: Battle of the network models: epilogue. J Water Resour Plan Manag 1987, 113:191-203.

Wu W, Simpson AR, Maier HR: Multi-objective genetic algorithm optimisation of water distribution systems accounting for sustainability. In Water Down Under 2008. Adelaide, Australia: Incorporating 31st Hydrology and Water Resources Symposium and the 4th International Conference on Water Resources and Environment Research (ICWRER); 2008a.

Wu W, Maier HR, Simpson AR: Genetic algorithm optimisation of water distribution systems accounting for greenhouse gas emissions. In 9th National Conference on Hydraulics in Water Engineering; 23-26 September. Darwin Convention Centre, Australia: Engineers Australia; 2008b.

Wu W, Simpson AR, Maier HR: Water distribution system optimisation accounting for a range of future possible carbon prices. In 10th Annual Symposium on Water Distribution Systems Analysis; 17-20 August. Kruger National Park, South Africa: American Society of Civil Engineers; 2008c.

Wu W, Maier HR, Simpson AR: Multi-objective water distribution system optimisation accounting for carbon emissions under different electricity tariffs. In Ozwater '09; March 16-18. Melbourne, Australia: Australian Water Association; 2009.

Wu W, Simpson AR, Maier HR: Accounting for greenhouse gas emissions in multiobjective genetic algorithm optimization of water distribution systems. J Water Resour Plan Manag 2010a, 136:146-155. 
Wu W, Simpson AR, Maier HR: Reducing carbon footprint from water distribution systems by using a lower discount rate. In Practical Responses to Climate Change National Conference 2010; 29 September-1 October. Melbourne, Australia: Engineers Australia; 2010b.

Wu W, Maier HR, Simpson AR: Single-objective versus multiobjective optimization of water distribution systems accounting for greenhouse gas emissions by carbon pricing. J Water Resour Plan Manag 2010c, 136:1-11.

Wu W, Simpson AR, Maier HR: Accounting for carbon: Selecting the appropriate discount rate for multi-objective optimisation of water distribution systems. In Ozwater '10; March 08-10. Brisbane, Australia: Australia Water Association; 2010d.

Wu W, Maier HR, Simpson AR: Sensitivity of optimal tradeoffs between cost and greenhouse gas emissions for water distribution systems to electricity tariff and generation. J Water Resour Plan Manag 2012a, 138:182-186

Wu W, Simpson AR, Maier HR, Marchi A: Incorporation of variable-speed pumping in multiobjective genetic algorithm optimization of the design of water transmission systems. J Water Resour Plan Manag 2012b, 138:543-552.

Wu W, Maier HR, Simpson AR: Multiobjective optimization of water distribution systems accounting for economic cost, hydraulic reliability, and greenhouse gas emissions. Water Resour Res 2013, 49:1211-1225.

Young J: The 'greening' of water: taking aim at climate change through reducing greenhouse gas emissions, increasing efficiency. J Am Water Works Assoc 2010, 102:20-22.

doi:10.1186/2194-6434-1-9

Cite this article as: Stokes et al:: The cost-greenhouse gas emission nexus for water distribution systems including the consideration of energy generating infrastructure: an integrated conceptual optimization framework and review of literature. Earth Perspectives 2014 1:9.

\section{Submit your manuscript to a SpringerOpen ${ }^{\circ}$ journal and benefit from:}

- Convenient online submission

- Rigorous peer review

- Immediate publication on acceptance

- Open access: articles freely available online

- High visibility within the field

- Retaining the copyright to your article

Submit your next manuscript at $\gg$ springeropen.com 